\title{
A serological assay to detect SARS-CoV-2 seroconversion in humans
}

\author{
Fatima Amanat $\oplus^{1,2}$, Daniel Stadlbauer ${ }^{1}$ ', Shirin Strohmeier ${ }^{1,3}$, Thi H. O. Nguyen $\oplus^{4}$, \\ Veronika Chromikova', Meagan McMahon', Kaijun Jiang1', Guha Asthagiri Arunkumar $\circledast^{1,2}$, \\ Denise Jurczyszak ${ }^{1,2}$, Jose Polanco 1,5, Maria Bermudez-Gonzalez', Giulio Kleiner ${ }^{1}$ ', Teresa Aydillo, ${ }^{1,6}$, \\ Lisa Miorin ${ }^{16}$, Daniel S. Fierer ${ }^{7}$, Luz Amarilis Lugo7, Erna Milunka Kojic ${ }^{7}$, Jonathan Stoever ${ }^{8}$, \\ Sean T. H. Liu ${ }^{1,7,910}$, Charlotte Cunningham-Rundles ${ }^{11,12}$, Philip L. Felgner ${ }^{13}$, Thomas Moran', \\ Adolfo García-Sastre ${ }^{10,6,7,14}$, Daniel Caplivski ${ }^{15}$, Allen C. Cheng ${ }^{16}$, Katherine Kedzierska ${ }^{4}{ }^{4}$, \\ Olli Vapalahti ${ }^{17,18,19}$, Jussi M. Hepojoki $\oplus^{17,20}$, Viviana Simon ${ }^{1,6,7}$ and Florian Krammer ${ }^{1 凶}$
}

\begin{abstract}
Here, we describe a serological enzyme-linked immunosorbent assay for the screening and identification of human SARS-CoV-2 seroconverters. This assay does not require the handling of infectious virus, can be adjusted to detect different antibody types in serum and plasma and is amenable to scaling. Serological assays are of critical importance to help define previous exposure to SARS-CoV-2 in populations, identify highly reactive human donors for convalescent plasma therapy and investigate correlates of protection.

Severeacute respiratorysyndrome coronavirus2 (SARS-CoV-2) a member of the subgenus Sarbecovirus - has spread globally, causing a pandemic with, so far, 3.6 million infections and 250,000 fatalities (as of 5 May 2020).

Nucleic acid tests that detect the SARS-CoV-2 RNA genome are now widely employed to diagnose coronavirus disease 2019 (COVID-19). However, there remains a great need for assays that measure antibody responses and determine seroconversion. While such serological assays are not well suited to detect acute infections, they support a number of highly relevant applications. First, serological assays allow us to study the immune response(s) to SARS-CoV-2 in a qualitative and quantitative manner. Second, serosurveys are needed to determine the precise rate of infection in an affected area, which is an essential variable to accurately determine the infection fatality rate. Third, serological assays will allow for the identification of individuals who mounted strong antibody responses and who could serve as donors for the generation of convalescent serum/plasma therapeutics. Lastly, serological assays
\end{abstract}

can help inform studies that aim to identify antibody responses that correlate with protection from SARS-CoV-2.

Sarbecoviruses express a large (approximately $140 \mathrm{kDa}$ ) glycoprotein termed spike protein (S, a homotrimer), which mediates binding to host cells via interactions with the human receptor angiotensin converting enzyme 2 (ACE2) ${ }^{1-3}$. The S protein is highly immunogenic with the receptor-binding domain (RBD) being the target of many neutralizing antibodies ${ }^{4}$. Individuals infected with coronaviruses typically mount neutralizing antibodies ${ }^{5}$ and a neutralizing response has been demonstrated for SARS-CoV-2 in an individual case from day 9 onwards $^{6}$. For human coronaviruses these responses have been linked to protection for a period of time and future studies will show if there is a correlation between neutralizing antibodies and protection from SARS-CoV-2 infection as well ${ }^{5}$. Serum neutralization can be measured using replication competent virus but the process requires several days and must be conducted in a biosafety level 3 laboratory for containment of SARS-CoV-2. Potentially, pseudotyped viral particle based entry assays using lentiviruses or vesicular stomatitis virus could be used but these reagents are not trivial to produce. A simple solution is the use of a binding assay, e.g. an enzyme-linked immunosorbent assays (ELISA), with recombinant antigen as substrate, especially if ELISA results correlate with neutralization assay results. Here we report the development of such an assay and provide a protocol for both recombinant antigen production as well as the ELISA methodology?

We generated two different versions of the SARS-CoV-2 spike protein, based on the genomic sequence of the first virus isolate,

\footnotetext{
'Department of Microbiology, Icahn School of Medicine at Mount Sinai, New York, NY, USA. ${ }^{2}$ Graduate School of Biomedical Sciences, Icahn School of Medicine at Mount Sinai, New York, NY, USA. ${ }^{3}$ Department of Biotechnology, University of Natural Resources and Life Sciences, Vienna, Austria. ${ }^{4}$ Department of Microbiology and Immunology, The Peter Doherty Institute for Infection and Immunity, University of Melbourne, Melbourne, Victoria, Australia. ${ }^{5}$ Department of Genetics and Genomic Sciences, Icahn School of Medicine at Mount Sinai, New York, NY, USA. ${ }^{6}$ Global Health and Emerging Pathogens Institute, Icahn School of Medicine at Mount Sinai, New York, NY, USA. 'Division of Infectious Diseases, Department of Medicine, Icahn School of Medicine at Mount Sinai, New York, NY, USA. ${ }^{8}$ Division of Pulmonary, Critical Care, and Sleep Medicine, Icahn School of Medicine at Mount Sinai, New York, NY, USA. ${ }^{\circ}$ Department of Medicine, Mount Sinai Queens, Astoria, NY, USA. ${ }^{10}$ Division of Hospital Medicine, Mount Sinai Health System, New York, NY, USA. "Division of Clinical Immunology, Department of Medicine, Icahn School of Medicine at Mount Sinai, New York, NY, USA. ${ }^{12}$ Department of Pediatrics, the Icahn School of Medicine at Mount Sinai, NY, USA. ${ }^{13}$ Department of Physiology and Biophysics, University of California, Irvine, Irvine, CA, USA. ${ }^{14}$ Tisch Cancer Institute, Icahn School of Medicine at Mount Sinai, New York, NY, USA. ${ }^{15}$ Travel Medicine Program, Division of Infectious Diseases, Icahn School of Medicine at Mount Sinai, New York, NY, USA. ${ }^{16}$ School of Public Health and Preventive Medicine, Monash University and Infection Prevention and Healthcare Epidemiology Unit Alfred Health, Melbourne, Victoria, Australia. ${ }^{17}$ Department of Virology, Medicum, University of Helsinki, Helsinki, Finland. ${ }^{18}$ Veterinary Biosciences, Veterinary Faculty, University of Helsinki, Helsinki, Finland. ${ }^{19}$ Department of Virology and Immunology, Helsinki University Hospital (HUSLAB), Helsinki, Finland. ${ }^{2}$ Institute of Veterinary Pathology, Vetsuisse Faculty, University of Zürich, Zürich, Switzerland. ${ }_{e}$-mail: florian.krammer@mssm.edu
} 

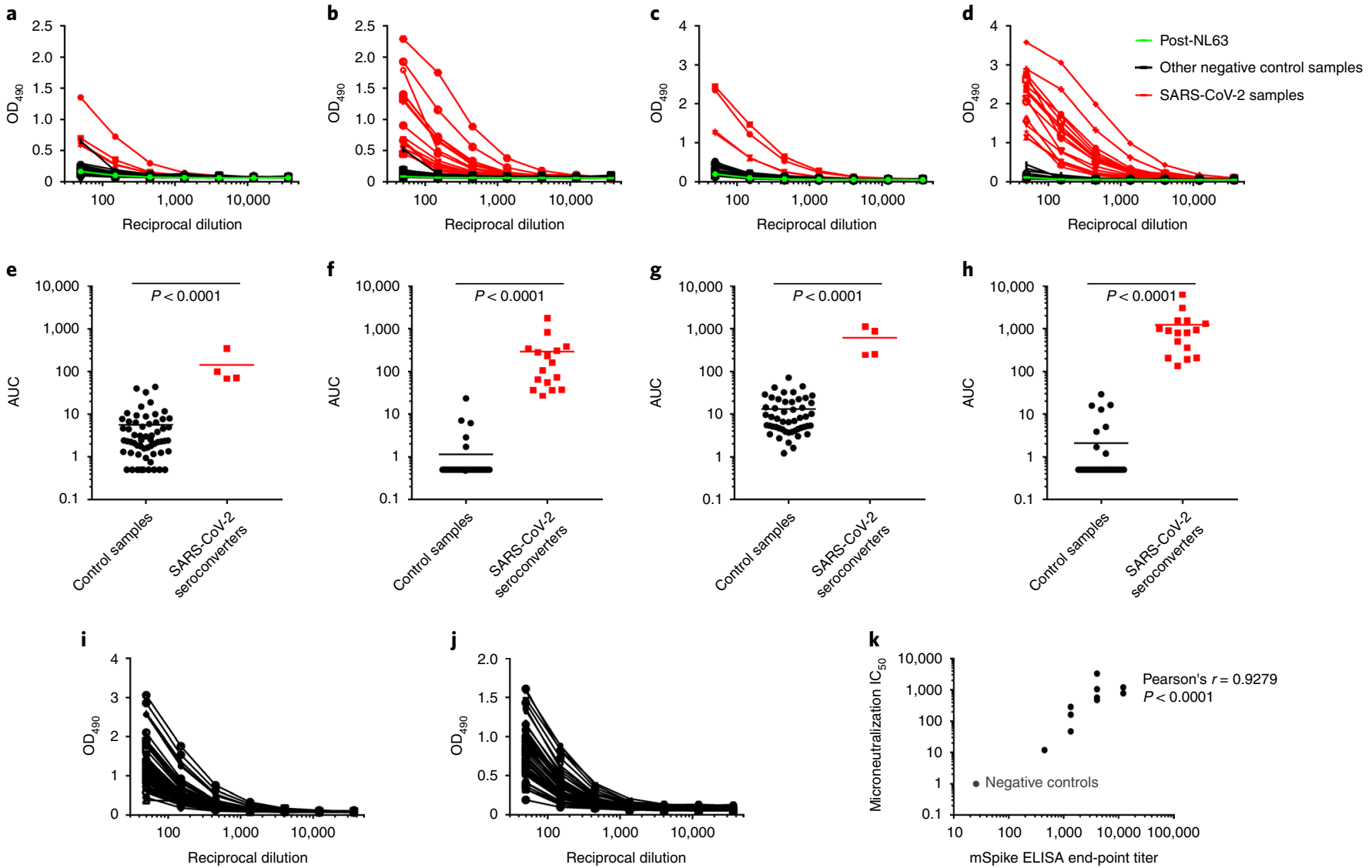

Fig. 1 Reactivity of control and SARS-CoV-2 convalescent sera to different spike antigens. a-d, Reactivity to iRBD (a), mRBD (b), iSpike (c) and mSpike (d). Red, green and black data points/lines show the results for sera from SARS-CoV-2-infected individuals, a convalescent serum sample post-NL63 infection and other negative control samples, respectively. e-h, Data from the same experiment as in a-d, respectively, but plotted as AUCs to obtain a better quantitative impression (control samples: $n=50$ for iRDB, iSpike and mSpike; $n=59$ for mRBD; convalescent samples: $n=4$ for iRBD and iSpike; $n=16$ for $m R B D$ and mSpike). Statistical analyses were performed using an unpaired two-tailed Student's t-test in GraphPad Prism. Horizontal lines represent mean values. $\mathbf{i}, \mathbf{j}$, Reactivity of the 50 negative control samples from $\mathbf{a}-\mathbf{h}$ against spike protein from human coronaviruses 229E (i) and NL63 (j). $\mathbf{k}$, Correlation between ELISA titers and microneutralization titers $(n=12$; the three samples from negative control sera overlap and are displayed as a single point). Statistical analysis was performed using Pearson's rank test in GraphPad Prism. The experiments were performed once. IC50, half-maximum inhibitory concentration.

Wuhan-Hu-1 ( ref. $^{8}$ ). The first construct encodes a full-length trimeric and stabilized version of the spike protein, whereas the second produces only the much smaller RBD. Sequences were codon optimized for mammalian cell expression. The full-length spike protein sequence was modified to remove the polybasic cleavage site, which is recognized by furin, and to add a pair of stabilizing mutations (Extended Data Fig. 1) $)^{2,9,10}$. These two modifications were included to enhance the stability of the protein based on published literature $^{2,9}$. At amino acid P1213, the sequence was fused to a thrombin cleavage site, a T4 foldon sequence for proper trimerization and a carboxy (C)-terminal hexahistidine tag for purification (Extended Data Fig. 1). The sequence was cloned into a pCAGGS vector for expression in mammalian cells and into a modified pFastBac Dual vector for the generation of baculoviruses and expression in insect cells. For expression of the RBD, the natural amino-terminal signal peptide of $\mathrm{S}$ was fused to the RBD sequence (amino acids 319-541) and joined with a C-terminal hexahistidine $\operatorname{tag}^{11}$. The same vectors as for the full-length $\mathrm{S}$ protein were used to express the RBD. In mammalian cells (Expi293F), the RBD domain gave high yields (approximately 25-50 mgl-1 of culture), but expression was lower in insect cells (approximately $1.5 \mathrm{mgl}-1$ of culture). Clear single bands were visible when the recombinant RBD proteins were analyzed by reducing sodium dodecyl sulfate-polyacrylamide gel electrophoresis (SDS-PAGE), with the insect cell-derived protein
(iRBD) running slightly lower than the mammalian cell-derived protein (mRBD) (Extended Data Fig. 1). The size difference probably reflects differences in glycan sizes between insect cells and mammalian cells. The full-length $\mathrm{S}$ protein was also expressed in both systems with higher yields in mammalian cells (mSpike) than in insect cells (iSpike) ( $~ 5$ versus $\sim 0.5 \mathrm{mgl}-1$ of culture). Reducing SDS-PAGE showed the full-length protein as a prominent band between 135 and $190 \mathrm{kDa}$, followed by a faint second band slightly below, which may be a cleavage product.

ELISAs were performed by serial dilution of the individual serum samples. Values from the dilution curves were used to determine the area under the curve (AUC), which was plotted on a graph. Initially, we tested a panel of 50 (59 for mRBD) banked human serum samples collected from study participants with and without confirmed previous viral infections (but otherwise healthy), to establish an ELISA with our proteins. These human sera were used to test the background reactivity to the SARS-CoV-2 spike in samples representative of the general US population from individuals ranging from 20 to $\geq 65$ years. An initial set of four plasma/serum samples from three COVID-19 survivors were used to determine the reactivity of SARS-CoV-2-infected individuals to the RBD and the full-length spike (Fig. 1).

All COVID-19 plasma/serum samples reacted strongly to both RBD and full-length spike protein, whereas reactivity of the 
other serum samples only yielded background reactivity (Fig. 1). Reactivity of COVID-19 sera was, in general, stronger against the full-length $S$ protein than against the RBD, both for raw optical density and AUC values, which may reflect the higher number of epitopes found on the much larger spike protein. For the RBD, the difference between control sera and convalescent samples (initial $n=4$ ) was larger when the mRBD was used compared with the iRBD. The same was true for the full-length spike protein. We tested an additional 12 serum samples from patients with acute COVID19 disease, as well as convalescent participants, for reactivity to mRBD and mSpike (Fig. 1). All 12 samples reacted with both RBD and spike protein. Thus, our assay distinguished sera from participants diagnosed with COVID-19 from serum samples collected before the pandemic (for example, collected in the autumn of 2019).

Our initial set of negative controls included convalescent serum from a participant with a confirmed NL63 infection. Importantly, this sample did not produce a signal against the SARS-CoV-2 RBD or spike. Since human coronaviruses OC43, 229E, NL63 and/or HKU1 are responsible for a large proportion of common colds every year, cross-reactivity between SARS-CoV-2 and these seasonal coronaviruses is of particular importance and warrants further investigation. To test how common antibodies to human coronaviruses other than SARS-CoV-2 are in our pre-pandemic serum panel, we performed ELISAs coated with spike protein of coronaviruses 229E and NL63. While none of the negative control sera reacted to SARS-CoV-2 RBD and spike, the majority of samples yielded strong signals to the spike proteins of these two human coronaviruses (Fig. 1i,j). In addition, we tested 21 different batches (27 vials) of pools of different products of normal human immune globulin (NHIG) that were intended for intravenous use and derived from $>1,000$ donors each. None of the NHIG preparations reacted with SARS-CoV-2 RBD or spike protein and the signal obtained was similar to that of the three irrelevant human monoclonal antibodies. In contrast, the RBD-binding monoclonal antibody CR3022 produced a strong signal in the ELISA (Extended Data Fig. 2a,b) ${ }^{12-14}$. Lastly, we tested a panel of 50 plasma samples collected from patients positive for human immunodeficiency virus and banked from 2008 and 2011. Again, none of the samples reacted with the SARS-CoV-2 RBD or spike (Extended Data Fig. 2c,d).

For the plasma/sera of patients with COVID-19 from our initial panel, we performed an isotyping and subtyping ELISA using the mammalian cell-expressed $S$ proteins. Strong reactivity was found for all samples for immunoglobulin G3 (IgG3), IgM and IgA (Extended Data Fig. 3a). An IgG1 signal was detected for the majority of samples, in addition to low reactivity for IgG2 (in five of the COVID-19 samples) and IgG4 (in four samples). Furthermore, we correlated the ELISA reactivity with the neutralizing activity of sera against the USA-WA1/2020 isolate. ELISA titers and microneutralization titers correlated significantly (Fig. 1k and Extended Data Fig. 3b), with a Spearman's r of 0.9279 ( $\mathrm{P}<0.0001)$.

One complexity with measuring antibodies in the bodily fluids of patients with COVID-19 is that infectious virus could be present in the biospecimen. To limit this risk, serum or plasma is heat inactivated for $1 \mathrm{~h}$ at $56^{\circ} \mathrm{C}$. To test whether such a heat treatment has an effect on detecting antibodies to the SARS-CoV-2 RBD and spike, we compared the reactivity of matched non-treated and heat-treated serum samples from patients with COVID-19. While slight differences were observed, they were minimal, suggesting that heat treatment may have no negative impact on assay performance (Fig. 2a,b). Similarly, we tested matched serum and plasma samples from patients with COVID-19 and found negligible differences, suggesting that both types of specimens can be used in the assay interchangeably (Fig. 2c,d).

Here, we describe a serological method to detect seroconversion upon SARS-CoV-2 infection. The method is based on reactivity to the immunogenic $S$ protein of the virus, is relatively simple
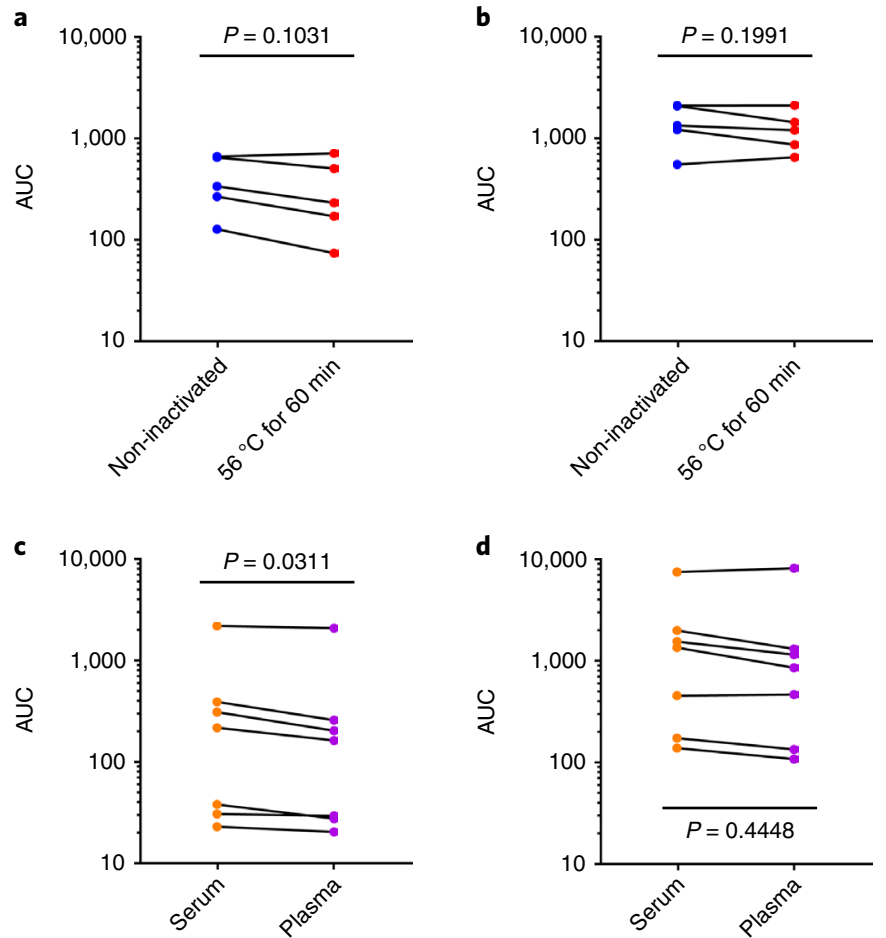

Fig. 2 | Effect of heat treatment and serum versus plasma on assay performance. $\mathbf{a}, \mathbf{b}$, Reactivity of paired non-treated serum and heat-treated serum samples to mRBD (a) and mSpike (b) of SARS-CoV-2 $(n=5)$. c,d, Reactivity of paired serum and plasma samples to mRBD (c) and mSpike (d) of SARS-CoV-2 $(n=7)$. Statistical analyses were performed using a paired two-tailed Student's t-test in GraphPad Prism. The experiments were performed once.

and quick in its execution and can be performed at biosafety level 2 as it does not involve live virus. We have tested this method using banked serum samples and NHIG preparations obtained from individuals before SARS-CoV-2 started to widely circulate in the United States. These serum samples produced low, close-to-baseline signals in our ELISAs. The age of the participants ranged from 20 to $\geq 65$ years of age and it is likely that most of these individuals had experienced infections with human coronaviruses, including the alphacoronaviruses NL63 and 229E, as well as the betacoronaviruses OC43 and HKU1 (ref. ${ }^{5}$ ). In fact, the majority of our negative control subjects had strong reactivity to the spike protein of NL63 and 229E, but showed no cross-reactivity to SARS-CoV-2 RBD and spike. We also included a convalescent serum sample from a participant with a laboratory-confirmed coronavirus NL63 infection. Our data show that there is no or only negligible cross-reactivity from human coronaviruses to SARS-CoV-2 in these individuals. Similar findings were reported in a recent study where sera from negative control subjects reacted well with spike proteins from human coronavirus but not with SARS-CoV-2 (ref. ${ }^{15}$ ). This is notable because it suggests that humans are serologically naive to SARS-CoV-2, which may explain the relatively high basic reproduction number (or R0) of SARS-CoV-2 compared with that of other respiratory viruses, such as influenza virus ${ }^{16}$. As a caveat, the reactivity of samples from SARS-CoV-1- or Middle East respiratory syndrome coronavirus-infected individuals was not tested, and cross-reaction might occur in this assay. Another caveat is of course the relatively small number of samples tested.

Our data show strong seroconversion with ELISA AUC values in the 1:1,000 range after natural infection with SARS-CoV-2. The results from our assay suggest that antibodies mounted upon 
infection target the full-length S protein as well as the RBD, which is the major target for neutralizing antibodies for related coronaviruses $^{4}$. In fact, one of the SARS-CoV-2 samples was previously tested in another study in neutralization assays and showed a neutralizing titer of 1:160 (ref. ${ }^{6}$ ). In addition, we performed microneutralization assays with a subset of our samples and found excellent correlation between our ELISA titers against the spike protein and virus neutralization, with several samples showing strong neutralizing activity with $50 \%$ inhibitory concentrations in the hundreds and thousands. This is in line with findings by Okba and colleagues ${ }^{17}$ who also found a strong correlation between ELISA and neutralization. Of note, the ELISA reagents used were derived from the original sequence from Wuhan, the neutralization assays were performed with USA-WA1/2020 (an Asian-lineage strain) and the majority of sera were obtained from subjects infected with European-lineage viruses $^{18}$. The observed correlation between ELISA and neutralization assays hints at minimal antigenic changes.

We believe that our ELISA method will be very useful for serosurveys aimed at determining the real attack rate and infection fatality rate in different human populations, and to map the kinetics of the antibody response to SARS-CoV-2. While we found seroconversion in severe, mild and asymptomatic cases, it is possible that some individuals do not seroconvert or that antibody titers wane within short periods of time. To be able to interpret serosurveys correctly, studies to assess the kinetics of the antibody response and the rate of non-responders are urgently needed. Clinical trials with convalescent serum as the therapeutic have been initiated in China (for example, NCT04264858). In addition, a recent report suggests that compassionate use of these interventions could be successful ${ }^{19}$. Screening potential plasma donors for high-antibody titers using our assay is faster and easier than performing standard neutralization assays in BSL3 containment laboratories. Our assay has already been implemented for this purpose in Mount Sinai's Clinical Laboratory Improvement Amendments-regulated clinical laboratory and has received emergency use authorization from New York State and from the Food and Drug Administration. Indeed, more than 250 patients with COVID-19 have been compassionately treated at Mount Sinai Hospital with antibody-rich plasma from convalescent donors identified with our assays (N. Bouvier, personal communication). Importantly, the assumption that antibodies to SARS-CoV-2 confer protection from reinfection needs to be confirmed and studies to investigate antibody titer as a correlate of protection should be started as soon as possible. We are making the methods and laboratory reagents widely available to the research community to support the global effort to limit and mitigate the spread of SARS-CoV-2.

\section{Online content}

Any methods, additional references, Nature Research reporting summaries, source data, extended data, supplementary information, acknowledgements, peer review information; details of author contributions and competing interests; and statements of data and code availability are available at https://doi.org/10.1038/s41591020-0913-5.

Received: 19 March 2020; Accepted: 28 April 2020;

Published online: 12 May 2020

\section{References}

1. Letko, M., Marzi, A. \& Munster, V. Functional assessment of cell entry and receptor usage for SARS-CoV-2 and other lineage B betacoronaviruses. Nat. Microbiol. 5, 562-569 (2020)

2. Wrapp, D. et al. Cryo-EM structure of the 2019-nCoV spike in the prefusion conformation. Science 367, 1260-1263 (2020).

3. Walls, A. C. et al. Structure, function, and antigenicity of the SARS-CoV-2 spike glycoprotein. Cell 181, 281-292 (2020).

4. Berry, J. D. et al. Neutralizing epitopes of the SARS-CoV S-protein cluster independent of repertoire, antigen structure or mAb technology. MAbs 2, 53-66 (2010).

5. Huang, A. T. et al. A systematic review of antibody mediated immunity to coronaviruses: antibody kinetics, correlates of protection, and association of antibody responses with severity of disease. Preprint at medRxiv https://doi.org/10.1101/2020.04.14.20065771 (2020).

6. Haveri, A. et al. Serological and Molecular Findings During SARS-CoV-2 Infection: the First Case Study in Finland, January to February 2020 (Eurosurveillance, 2020).

7. Stadlbauer, D. et al. SARS-CoV-2 seroconversion in humans: a detailed protocol for a serological assay, antigen production, and test setup. Curr. Protoc. Microbiol. 57, e100 (2020).

8. Wu, F. et al. A new coronavirus associated with human respiratory disease in China. Nature 579, 265-269 (2020).

9. Pallesen, J. et al. Immunogenicity and structures of a rationally designed prefusion MERS-CoV spike antigen. Proc. Natl Acad. Sci. USA 114, E7348-E7357 (2017).

10. Kirchdoerfer, R. N. et al. Pre-fusion structure of a human coronavirus spike protein. Nature 531, 118-121 (2016).

11. Li, F., Li, W., Farzan, M. \& Harrison, S. C. Structure of SARS coronavirus spike receptor-binding domain complexed with receptor. Science 309, 1864-1868 (2005).

12. Tian, X. et al. Potent binding of 2019 novel coronavirus spike protein by a SARS coronavirus-specific human monoclonal antibody. Emerg. Microbes Infect. 9, 382-385 (2020)

13. Ter Meulen, J. et al. Human monoclonal antibody combination against SARS coronavirus: synergy and coverage of escape mutants. PLoS Med. 3, e237 (2006).

14. Yuan, M. et al. A highly conserved cryptic epitope in the receptor-binding domains of SARS-CoV-2 and SARS-CoV. Science 3, eabb7269 (2020).

15. Khan, S. et al. Analysis of serologic cross-reactivity between common human coronaviruses and SARS-CoV-2 using coronavirus antigen microarray. Preprint at bioRxiv https://doi.org/10.1101/2020.03.24.006544 (2020).

16. Li, Q. et al. Early transmission dynamics in Wuhan, China, of novel coronavirus-infected pneumonia. N. Engl. J. Med. 26, 1199-1207 (2020).

17. Okba, N. M. A. et al. Severe acute respiratory syndrome coronavirus 2 -specific antibody responses in coronavirus disease 2019 patients. Emerg. Infect. Dis. https://doi.org/10.3201/eid2607.200841 (2020).

18. Gonzalez-Reiche, A. S. et al. Introductions and early spread of SARS-CoV-2 in the New York City area. Preprint at medRxiv https://doi.org/10.1101/ 2020.04.08.20056929 (2020).

19. Shen, C. et al. Treatment of 5 critically ill patients with COVID-19 with convalescent plasma. J. Am. Med. Assoc. 232, 1582-1589 (2020).

Publisher's note Springer Nature remains neutral with regard to jurisdictional claims in published maps and institutional affiliations.

(C) The Author(s), under exclusive licence to Springer Nature America, Inc. 2020 


\section{Methods}

Recombinant proteins. The mammalian cell codon-optimized nucleotide sequence coding for the spike protein of the SARS-CoV-2 isolate (GenBank: MN908947.3) was synthesized commercially (Genewiz). The RBD (amino acids 319-541; RVQP...CVNF), along with the signal peptide (amino acids 1-14; MFVF...VSSQ) plus a hexahistidine tag, was cloned into mammalian expression vector pCAGGS as well as in a modified pFastBac Dual vector for baculovirus system expression. The soluble version of the spike protein (amino acids 1-1,213; MFVF...IKWP), including a C-terminal thrombin cleavage site, T4 foldon trimerization domain and hexahistidine tag, was also cloned into pCAGGS. The protein sequence was modified to remove the polybasic cleavage site (RRAR to A), and two stabilizing mutations were introduced as well (K986P and V987P; wild-type numbering). Recombinant proteins were produced using the well-established baculovirus expression system and this system has been published in detail in refs. ${ }^{20-22}$, including a video guide. Recombinant proteins were also produced in Expi293F cells (Thermo Fisher Scientific) by transfections of these cells with purified DNA using an ExpiFectamine 293 Transfection Kit (Thermo Fisher Scientific). Supernatants from transfected cells were harvested on day 3 post-transfection by centrifugation of the culture at $4,000 \mathrm{~g}$ for $20 \mathrm{~min}$. Supernatant was then incubated with $6 \mathrm{ml} \mathrm{Ni}$-NTA Agarose (Qiagen) for $1-2 \mathrm{~h}$ at room temperature. Next, gravity flow columns were used to collect the Ni-NTA agarose and the protein was eluted. Each protein was concentrated in Amicon centrifugal units (EMD Millipore) and re-suspended in phosphate-buffered saline (PBS). Proteins were analyzed by reducing SDS-PAGE. The DNA sequence for all constructs is available from the Krammer Laboratory and has also been deposited in GenBank (additional information in the 'Data availability' statement). Several of the expression plasmids and proteins have also been submitted to the BEI Resources repository and can be requested from their web page for free (https:// www.beiresources.org/). S1 proteins of NL63 and 229E were obtained from Sino Biological (produced in hexahistidine-tagged 293HEK cells). A detailed protocol for protein expression of RBD and spike in mammalian cells is also available ${ }^{7}$.

SDS-PAGE. Recombinant proteins were analyzed via a standard SDS-PAGE gel to check protein integrity. Protein $(1 \mu \mathrm{g})$ was mixed with $2 \times$ Laemmli buffer containing $5 \% \beta$-mercaptoethanol at a ratio of $1: 1$. Samples were heated at $100^{\circ} \mathrm{C}$ for $15 \mathrm{~min}$ and then loaded onto a polyacrylamide gel (5-20\% gradient; Bio-Rad). Gels were stained with SimplyBlue SafeStain (Invitrogen) for $1-2 \mathrm{~h}$ and then de-stained in distilled water overnight.

\section{Human samples. Human plasma and serum samples were obtained from a} number of different sources.

First, de-identified samples from the University of Melbourne $(n=3$; taken on days 2, 4 and 6 after symptom onset) and University of Helsinki ( $\mathrm{n}=1$; taken on day 20 after symptom onset) (neutralizing titer: $1: 160)^{6}$ were used as positive controls. For these, human experimental work was conducted according to the Declaration of Helsinki principles and according to the Australian National Health and Medical Research Council Code of Practice. All participants provided written informed consent before the study. The studies were approved by the Alfred Hospital (ID number 280/14) and University of Melbourne (ID numbers 1442952.1 and 1955465.2) Human Research Ethics Committees, and under a research permit for project TYH2018322 of Helsinki University Hospital Laboratory.

Second, banked human samples were collected from study participants enrolled in several ongoing Institutional Review Board-approved longitudinal observational study protocols of the Mount Sinai Personalized Virology Initiative. The pre-pandemic serum panel comprised samples selected based on the date of collection (for example, autumn 2019) and whether participants had a documented history of viral infection (for example, dengue virus, hantavirus, Chikungunya virus or coronavirus NL63). All participants agreed to sample banking and future research use. Self-reported ethnicities of the individuals from whom samples were tested included Caucasian, Asian, African American and Hispanic. Samples included convalescent sera from a participant with an NL63 infection, as determined by the BioFire Respiratory Panel. We included serum collected at day 3 post-symptom onset, as well as convalescent serum from the same person (day 30 post-symptom onset). These samples served as negative controls given that they were collected before SARS-CoV-2 spread in the United States. Six subjects were 20-29 years of age, 18 were 30-39 years of age, 13 were 40-49 years of age, seven were 50-59 years of age and six were 60 years or older. For the mRBD ELISAs, sera from an additional nine subjects were tested (two aged $30-39$ years, four aged $40-49$ years, two aged $50-59$ years and one aged $\geq 60$ years). The pre-pandemic panel was complemented by a panel of plasma samples collected between 2008 and 2011 from 50 individuals infected with human immunodeficiency virus 1 .

Third, the Mount Sinai COVID-19 panel comprised serum $(n=12)$ and plasma samples from individuals with severe, mild or asymptomatic SARS-CoV-2 infections (see Supplementary Table 1). Seven paired serum and plasma samples from patients with COVID-19 were used for comparison purposes. These samples were collected between 7 and $30 \mathrm{~d}$ post-symptom onset. These samples were collected from participants enrolled in an ongoing Institutional Review Board-approved study of the Mount Sinai Personalized Virology Initiative.
NHIG. The following NHIG preparations, each prepared from $>1,000$ blood or plasma donors and intended for intravenous use for medical conditions, were tested in an ELISA to determine whether they had reactivity against SARS-CoV-2 spike or RBD: Octagam (M934A8541); Gamunex-c (B2GMD00943, A1GLD01882, B3GLD01223, A1GLD01902, B2GLD01972, B3GGD00143 and A1GKE00012 (two different vials) and B2GKD00863 and B2GJE00033 (three different vials)); Gammagard Liquid (LE12T292AB, LE12V238AB and LE12V278AD); Gammagard S/D (LE08V027AB (four different vials)); and Gammagard Liquid (C19G080AAA, LE12V071AD, LE12V230AB, LE12V115AC, LE12V205AB, LE12VE25AB and LE12V115AC).

ELISA. The ELISA protocol was adapted from previously established protocols ${ }^{23,24}$. Overnight, 96-well plates (Immulon 4 HBX; Thermo Fisher Scientific) were coated at $4{ }^{\circ} \mathrm{C}$ with $50 \mu \mathrm{l}$ per well of a $2 \mu \mathrm{g} \mathrm{ml}-1$ solution of each respective protein suspended in PBS (Gibco). The next morning, the coating solution was removed and $100 \mu \mathrm{l}$ per well of $3 \%$ non-fat milk prepared in PBS with $0.1 \%$ Tween 20 (PBST) was added to the plates at room temperature for $1 \mathrm{~h}$ as a blocking solution. Serum samples were heated at $56^{\circ} \mathrm{C}$ for $1 \mathrm{~h}$ before use to reduce the risk from any potential residual virus in the serum. Serial dilutions of serum and antibody samples were prepared in $1 \%$ non-fat milk prepared in PBST. The blocking solution was removed and $100 \mu \mathrm{l}$ of each serial dilution was added to the plates for $2 \mathrm{~h}$ at room temperature. Next, the plates were washed three times with $250 \mu \mathrm{l}$ per well of $0.1 \%$ PBST. Next, a 1:3,000 dilution of goat anti-human IgG-horseradish peroxidase (HRP) conjugated secondary antibody (Thermo Fisher Scientific) was prepared in $0.1 \%$ PBST and $100 \mu$ of this secondary antibody was added to each well for $1 \mathrm{~h}$. Plates were again washed three times with $0.1 \%$ PBST. Once completely dry, $100 \mu$ l SIGMAFAST OPD (o-phenylenediamine dihydrochloride; SigmaAldrich) solution was added to each well. This substrate was left on the plates for $10 \mathrm{~min}$ and then the reaction was stopped by the addition of $50 \mu \mathrm{l}$ per well of $3 \mathrm{M}$ hydrochloric acid. The optical density at $490 \mathrm{~nm}$ (OD490) was measured using a Synergy 4 (BioTek) plate reader. The background value was set at an OD490 of 0.11 and the AUC was calculated. AUC values below 1 were assigned a value of 0.5 for plotting and calculation purposes. Data were analyzed using Prism 7 (GraphPad). In some cases, end-point titers were calculated, with the end-point titer being the last dilution before reactivity dropped below an OD490 of $<0.11$. To determine the impact of heat treatments, paired samples that were heat treated or not treated were analyzed. NHIGs were run similar to serum and plasma samples but with a starting dilution at a concentration of $100 \mu \mathrm{g} \mathrm{ml}-1$. Three non-SARS-CoV-2 reactive human monoclonal antibodies and CR3022 (refs. ${ }^{12-14}$ ) - a human monoclonal antibody reactive to the RBD of both SARS-CoV-1 and SARS-CoV-2-were used as controls.

To assess the distribution of the different antibody isotypes and subclasses in the samples that reacted well in our standard ELISA, another ELISA was performed with different secondary antibodies ${ }^{25}$. These antibodies included anti-human IgA ( $\alpha$-chain-specific) HRP antibody (Sigma-Aldrich; A0295; $1: 3,000)$, anti-human IgM ( $\mu$-chain-specific) HRP antibody (Sigma-Aldrich; A6907; 1:3,000), anti-human IgG1 Fc-HRP (Southern Biotech; 9054-05; 1:3,000), anti-human IgG2 Fc-HRP (Southern Biotech; 9060-05; 1:3,000), anti-human IgG3 hinge-HRP (Southern Biotech; 9210-05; 1:3,000) and anti-human IgG4 Fc-HRP (Southern Biotech; 9200-05; 1:3,000).

Of note, different ELISA substrates and stopping solutions that are less hazardous may be used in order to comply to local guidelines if appropriate.

Microneutralization assay. Vero E6 cells were seeded at a density of 20,000 cells per well in a 96-well cell culture plate in complete Dulbecco's Modified Eagle Medium (cDMEM). The following day, heat-inactivated serum samples (dilution of $1: 10)$ were serially diluted threefold in $1 \times$ MEM $(10 \% 10 \times$ minimal essential medium (Gibco), $2 \mathrm{mM}$ L-glutamine, $0.1 \%$ sodium bicarbonate (wt/vol; Gibco), $10 \mathrm{mM}$ 4-(2-hydroxyethyl)-1-piperazineethanesulfonic acid (HEPES; Gibco), $100 \mathrm{U} \mathrm{ml}^{-1}$ penicillin, $100 \mathrm{ug} / \mathrm{ml}^{-1}$ streptomycin (Gibco) and $0.2 \%$ bovine serum albumin (MP Biomedicals)). The authentic SARS-CoV-2 virus (USA-WA1/2020; GenBank: MT020880) was diluted to a concentration of $100 \mathrm{TCID}_{50}(50 \%$ tissue culture infectious dose) in $1 \times$ MEM. Then, $80 \mu \mathrm{l}$ of each serum dilution and $80 \mu \mathrm{l}$ of the virus dilution were added to a 96-well cell culture plate and allowed to incubate for $1 \mathrm{~h}$ at room temperature. cDMEM was removed from Vero E6 cells and $120 \mu \mathrm{l}$ of the virus-serum mixture was added to the cells. Then, the cells were incubated at $37^{\circ} \mathrm{C}$ for $1 \mathrm{~h}$. After the 1 -h incubation, the virus-serum mixture was removed from the cells and $100 \mu \mathrm{l}$ of each corresponding serum dilution and $100 \mu \mathrm{l}$ of $1 \times$ MEM containing $1 \%$ FBS (Corning) was added to the cells. The cells were incubated for $48 \mathrm{~h}$ at $37^{\circ} \mathrm{C}$ and then fixed with $10 \%$ paraformaldehyde (Polysciences) for $24 \mathrm{~h}$ at $4^{\circ} \mathrm{C}$. Following fixation, the paraformaldehyde was removed and the cells were washed with $200 \mu \mathrm{l}$ PBS. The cells were then permeabilized by the addition of $150 \mu \mathrm{l}$ PBS containing $0.1 \%$ Triton X-100 for $15 \mathrm{~min}$ at room temperature. The plates were then washed three times with PBS containing PBST and blocked in blocking solution (3\% milk (American Bio) in PBST) for $1 \mathrm{~h}$ at room temperature. After blocking, $100 \mu \mathrm{l}$ of mAb 1C7 (anti-SARS nucleoprotein antibody generated in house) at a dilution of 1:1,000 was added to all wells and the plates were allowed to incubate for $1 \mathrm{~h}$ at room temperature. The plates were then washed three times with PBST before the addition of goat 
anti-mouse IgG-HRP (Rockland Immunochemicals), diluted 1:3,000 in blocking solution for $1 \mathrm{~h}$ at room temperature. Plates were then washed three times with PBST, and SIGMAFAST OPD (Sigma-Aldrich) was added. After a 10-min incubation at room temperature, the reaction was stopped by adding $50 \mu \mathrm{l} 3 \mathrm{M}$ hydrochloric acid to the mixture. The OD 490 was measured on a Synergy 4 plate reader (BioTek). A cut-off value of the average of the optical density values of blank wells plus three standard deviations was established for each plate and used to calculate the microneutralization titer. Microneutralization assays were performed in a facility with a biosafety level of 3 .

Statistical analysis. Differences between negative controls and positive controls were analyzed using an unpaired t-test. Differences between paired non-treated and heat-treated samples, as well as paired serum and plasma samples, were analyzed using a paired t-test. Correlations between ELISA titers and neutralization titers were analyzed using Spearman's rank test. Analyses were performed with GraphPad Prism.

Reporting Summary. Further information on research design is available in the Nature Research Reporting Summary linked to this article.

\section{Data availability}

The data shown in the manuscript are available upon request from the corresponding author. Nucleotide sequences of both constructs have been submitted to NCBI (GenBank IDs MT380724.1 and MT380725.1). Expression plasmids have been deposited to BEI Resources (https://www.beiresources.org/), and plasmids and sequences are also available from the corresponding author. A detailed protocol for expression and ELISA setup has been published (https://currentprotocols.onlinelibrary.wiley.com/doi/full/10.1002/cpmc.100).

\section{References}

20. Amanat, F. et al. Antibodies to the glycoprotein GP2 subunit cross-react between Old and New World arenaviruses. mSphere 3, e00189-18 (2018).

21. Krammer, F. et al. A carboxy-terminal trimerization domain stabilizes conformational epitopes on the stalk domain of soluble recombinant hemagglutinin substrates. PLoS ONE 7, e43603 (2012).

22. Margine, I., Palese, P. \& Krammer, F. Expression of functional recombinant hemagglutinin and neuraminidase proteins from the novel H7N9 influenza virus using the baculovirus expression system. J. Vis. Exp. 6, e51112 (2013).

23. Amanat, F., Meade, P., Strohmeier, S. \& Krammer, F. Cross-reactive antibodies binding to $\mathrm{H} 4$ hemagglutinin protect against a lethal H4N6 influenza virus challenge in the mouse model. Emerg. Microbes Infect. 8, 155-168 (2019).

24. Wohlbold, T. J. et al. Broadly protective murine monoclonal antibodies against influenza B virus target highly conserved neuraminidase epitopes. Nat. Microbiol. 2, 1415-1424 (2017).

25. Rajendran, M. et al. Analysis of anti-influenza virus neuraminidase antibodies in children, adults, and the elderly by ELISA and enzyme inhibition: evidence for original antigenic sin. mBio 8, e02281-16 (2017).

\section{Acknowledgements}

We thank Y.-Z. Zhang (Fudan University) and E. Holmes (University of Sydney) for sharing the sequence of the first SARS-CoV-2 isolate in a very timely manner. We thank J. Garlick and J. Roney (Alfred Hospital, Melbourne) for data and specimen collection, N. Aboelregal for making many different NHIG products (Mount Sinai) available and L. Martinez-Sobrido (Texas Biomedical Research Institute) for initially characterizing $\mathrm{mAb} 1 \mathrm{C} 7$. We are also thankful to Genewiz for speeding up gene synthesis for this project, and for being very accommodating to our needs. Furthermore, we thank D. Tidmore for help with ordering primers quickly, and finally S. Dong for commuting to New Jersey on several occasions to pick up reagents from Genewiz. We also thank the Mount Sinai Health System Translational Science Hub 'ConduITS' (NIH grant U54TR001433) for supporting sample collection. The work of the Personalized Virology Initiative is supported by institutional funds and philanthropic donations. This work was partially supported by the NIAID Centers of Excellence for Influenza Research and Surveillance (CEIRS; contract HHSN272201400008C to F.K. and A.G.-S.), the Collaborative Influenza Vaccine Innovation Centers (CIVIC; contract 75N93019C00051 to F.K. and A.G.-S.), Open Philanthropy, the Australian National Health and Medical Research Council (NHMRC Program Grant 1071916 and NHMRC Research Fellowship Level B (1102792) to K.K.), the Academy of Finland to O.V. and J.M.H., as well as Jane and Aatos Erkko Foundation and Helsinki University Hospital Funds to O.V. Furthermore, we thank our generous community for providing essential funds and support for our SARS-CoV-2 and COVID-19 research efforts. The following reagent was deposited by the Centers for Disease Control and Prevention and obtained through BEI Resources, NIAID, NIH: SARS-Related Coronavirus 2, Isolate USA-WA1/2020, NR-52281. Finally, we thank all of the study participants for their contribution to the research. We wish the patients with COVID-19 a speedy recovery.

\section{Author contributions}

F.A., V.S. and F.K. conceived of and designed the study. F.A., D.S., S.S., T.H.O.N., V.C., M.M., K.J., G.A.A., D.J., J.P., M.B.-G., G.K., T.A., L.M., D.S.F., L.A.L., E.M.K., J.S., S.T.H.L., C.C.-R., P.L.F., A.G.-S., D.C., A.C.C., K.K., O.V. and J.M.H. collected data and contributed samples. F.A., V.S. and F.K. analyzed the data and wrote the manuscript.

\section{Competing interests}

Mount Sinai is in the process of licensing out assays to commercial entities based on the assays described here and has filed for patent protection.

\section{Additional information}

Extended data is available for this paper at https://doi.org/10.1038/s41591-020-0913-5.

Supplementary information is available for this paper at https://doi.org/10.1038/ s41591-020-0913-5.

Correspondence and requests for materials should be addressed to F.K.

Peer review information Alison Farrell is the primary editor on this article and managed its editorial process and peer review in collaboration with the rest of the editorial team.

Reprints and permissions information is available at www.nature.com/reprints. 
A

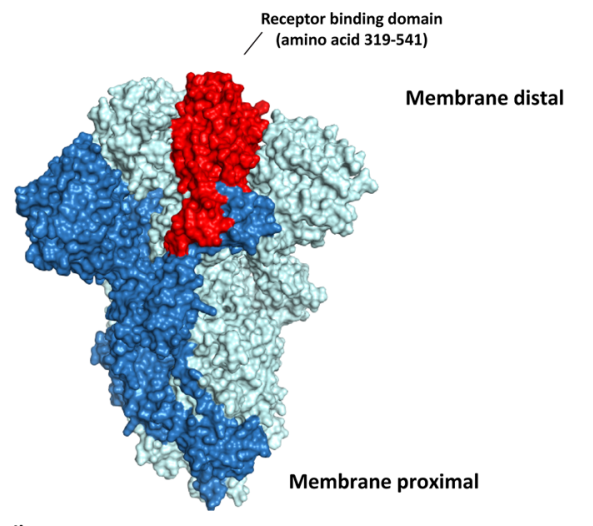

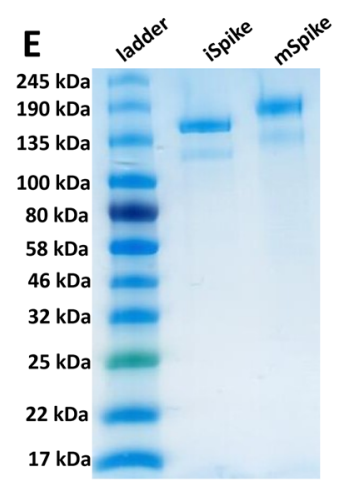

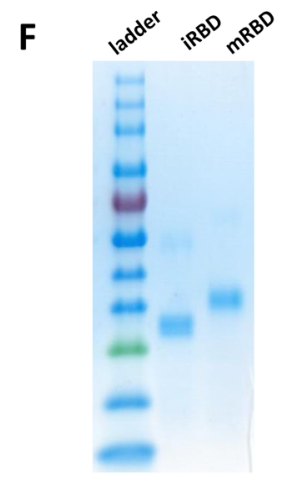

B

Wild type spike

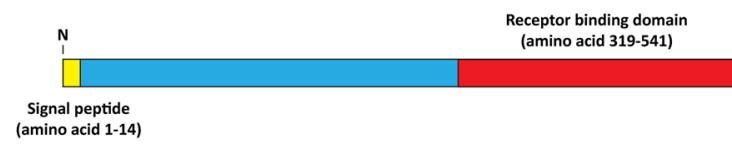

si

S1 $\underset{\text { (amino acid 682-685/RRAR) }}{\text { Polybasic cleavagite }} \rightarrow$ s2

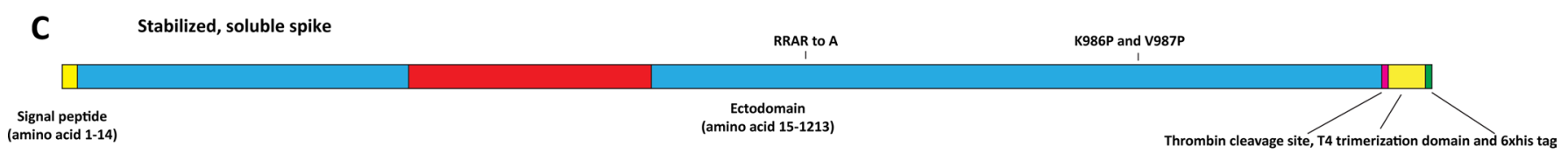

D Soluble receptor binding domain

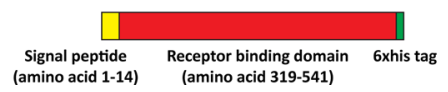

Extended Data Fig. 1 | Constructs for recombinant protein expression. a, Visualization of the trimeric spike protein of SARS-CoV-2 based on PBD \# 6VXX using $\mathrm{Pymol}^{3}$. One monomer is colored in dark blue while the remaining two monomers are held in light blue. The receptor binding domain (RBD) of the dark blue trimer is highlighted in red. $\mathbf{b}$, Schematic of the wild type full length spike protein with signal peptide, ectodomain, receptor binding domain, furin cleavage site, S1, S2, and transmembrane and endodomain domain indicated. c, Schematic of the soluble trimeric spike. The polybasic/furin cleavage site (RRAR) was replaced by a single A. The transmembrane and endodomain were replaced by a furin cleavage site, a T4 foldon tetramerization domain and a hexahistidine tag. Introduction of K986P and V987P has been shown to stabilize the trimer in the pre-fusion conformation. $\mathbf{d}$, Schematic of the soluble receptor binding domain construct. All constructs are to scale. e Reducing SDS PAGE of insect cell and mammalian cell derived soluble trimerized spike protein (iSpike and mSpike). $\mathbf{f}$ Reducing SDS PAGE of insect cell derived and mammalian cell derived recombinant receptor binding domain (iRBD and mRBD). Experiments were performed six times with the same result. 
A
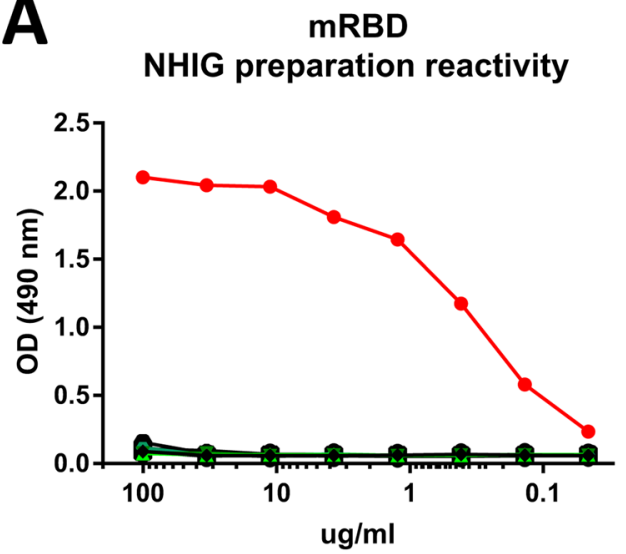

C reactivity of historic HIV+ samples

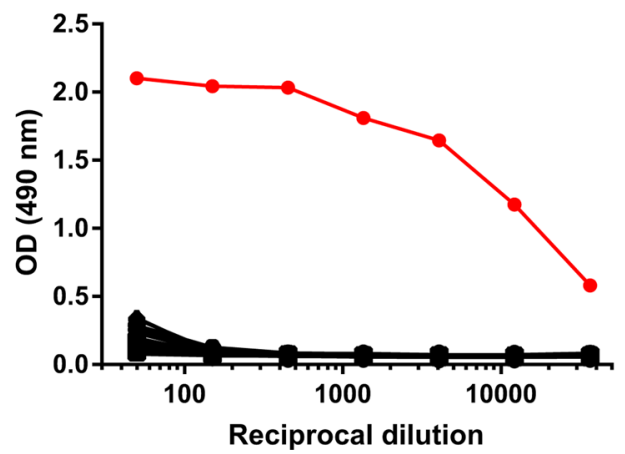

B

mSpike NHIG preparation reactivity

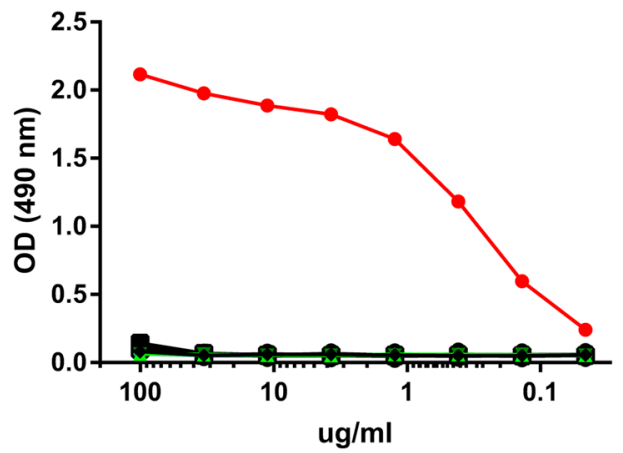

$\rightarrow$ NHIG preparations 1-27

- Neg. contr. mAb 1

$\rightarrow$ Neg. contr. mAb 2

- Neg. contr. mAb 3

$\multimap$ mAb CR3022

D

mSpike reactivity of historic HIV+ samples

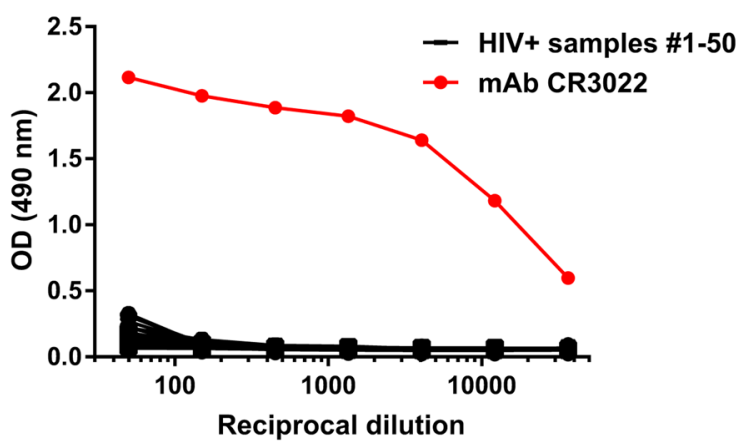

Extended Data Fig. 2 | Human normal immunoglobulin preparations and historic sera from HIV + patients do not react with the SAR-CoV-2 spike. $\mathbf{a}, \mathbf{b}$, Reactivity of 21 different pools of human normal immunoglobulin (HNIG) preparations (27 different vials) to mRBD and mSpike of SARS-CoV-2. MAb CR3022 was used as positive control, three different irrelevant human mAbs were used as negative control. $\mathbf{c}$, $\mathbf{d}$ shows reactivity of historic samples from $50 \mathrm{HIV}+$ individuals to $\mathrm{mRBD}$ and mSpike of SARS-CoV-2. Both HNIG and serum samples from HIV + donors were collected before the SARS-CoV-2 pandemic. Experiments were performed once. MAb CR3022 was used as positive control at a starting concentration of $100 \mathrm{ug} / \mathrm{ml}$. Of note, the experiments in $A$ and $C$ as well as $B$ and $D$ were done at the same time and their positive controls are shared and displayed in both panels. Experiments were performed once. 
A mSpike isotyping/subtyping

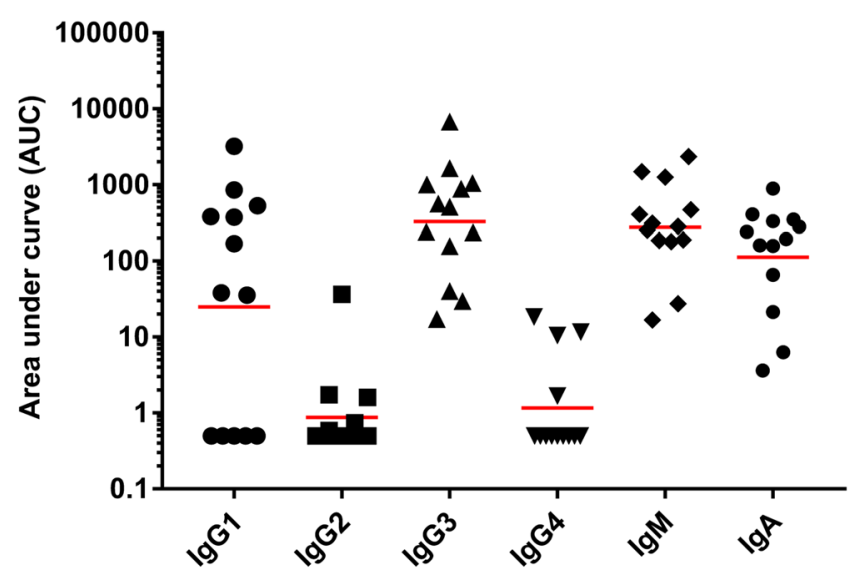

B

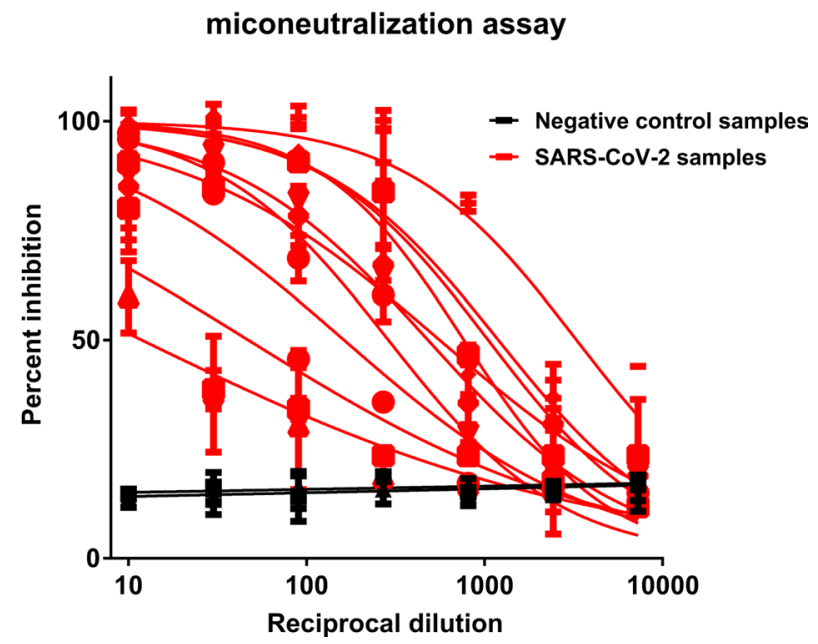

Extended Data Fig. 3 | Isotypes and subtypes of antibodies from COVID19 patients to the soluble spike protein and microneutralization titers. a, Mammalian cell derived spike protein was used to study isotype/subclass distribution of antibodies ( $n=13$ positive samples). Lines represent the geometric mean. $\mathbf{b}$, Microneutralization assay $(n=12)$ performed with authentic SARS-CoV-2. Lines represent curves fitted using an inhibitor $(\log )$ versus response variable slope with four parameters function in Graphpad Prism. Experiments were performed once. 


\section{Reporting Summary}

Nature Research wishes to improve the reproducibility of the work that we publish. This form provides structure for consistency and transparency in reporting. For further information on Nature Research policies, see Authors \& Referees and the Editorial Policy Checklist.

\section{Statistics}

For all statistical analyses, confirm that the following items are present in the figure legend, table legend, main text, or Methods section.

n/a Confirmed

\The exact sample size $(n)$ for each experimental group/condition, given as a discrete number and unit of measurement

Х $\square$ A statement on whether measurements were taken from distinct samples or whether the same sample was measured repeatedly

$\varnothing$ The statistical test(s) used AND whether they are one- or two-sided

Only common tests should be described solely by name; describe more complex techniques in the Methods section.

Х $\square$ A description of all covariates tested

$\triangle \square$ A description of any assumptions or corrections, such as tests of normality and adjustment for multiple comparisons

$\square$ A full description of the statistical parameters including central tendency (e.g. means) or other basic estimates (e.g. regression coefficient)

$\triangle \square$ AND variation (e.g. standard deviation) or associated estimates of uncertainty (e.g. confidence intervals)

For null hypothesis testing, the test statistic (e.g. $F, t, r$ ) with confidence intervals, effect sizes, degrees of freedom and $P$ value noted

Give $P$ values as exact values whenever suitable.

Х $\square$ For Bayesian analysis, information on the choice of priors and Markov chain Monte Carlo settings

Х $\square$ For hierarchical and complex designs, identification of the appropriate level for tests and full reporting of outcomes

$\bigotimes \square$ Estimates of effect sizes (e.g. Cohen's $d$, Pearson's $r$ ), indicating how they were calculated

Our web collection on statistics for biologists contains articles on many of the points above.

\section{Software and code}

\section{Policy information about availability of computer code}

\section{Data collection No software was used for data collection.}

Data analysis GraphPad Prism 7.05

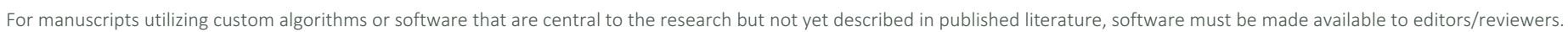
We strongly encourage code deposition in a community repository (e.g. GitHub). See the Nature Research guidelines for submitting code \& software for further information.

\section{Data}

Policy information about availability of data

All manuscripts must include a data availability statement. This statement should provide the following information, where applicable:

- Accession codes, unique identifiers, or web links for publicly available datasets

- A list of figures that have associated raw data

- A description of any restrictions on data availability

The data shown in the manuscript is available upon request from the corresponding author.

\section{Field-specific reporting}

Please select the one below that is the best fit for your research. If you are not sure, read the appropriate sections before making your selection.

$\bigotimes$ Life sciences $\quad \square$ Behavioural \& social sciences $\quad \square$ Ecological, evolutionary \& environmental sciences

For a reference copy of the document with all sections, see nature.com/documents/nr-reporting-summary-flat.pdf 


\section{Life sciences study design}

All studies must disclose on these points even when the disclosure is negative.

Sample size Sample size was determined/limited by available number of samples. The maximum number of samples available was analyzed. Positive and negative control samples for assay development showed a clear difference in reactivity that could already be detected with an $\mathrm{n}$ of 4 positive samples.

Data exclusions All data was included in the analysis

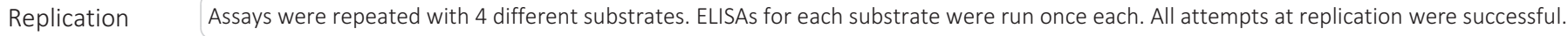

Randomization Randomization was not performed since the purpose of this work was assay development.

Blinding Blinding was not performed since the purpose of this work was assays development. Performance tests of this assay setup in our clinical laboratory have been conducted using blinded operators.

\section{Reporting for specific materials, systems and methods}

We require information from authors about some types of materials, experimental systems and methods used in many studies. Here, indicate whether each material, system or method listed is relevant to your study. If you are not sure if a list item applies to your research, read the appropriate section before selecting a response.

\begin{tabular}{|c|c|}
\hline $\mathrm{n} / \mathrm{a}$ & Involved in the study \\
\hline - & 冈 Antibodies \\
\hline L & $\bigotimes$ Eukaryotic cell lines \\
\hline$\bigotimes$ & $\square$ Palaeontology \\
\hline$\bigotimes$ & $\square$ Animals and other organisms \\
\hline Г & $\bigotimes$ Human research participants \\
\hline$\sqrt{ }$ & $\square$ Clinical data \\
\hline
\end{tabular}

\begin{tabular}{|c|c|}
\hline $\mathrm{n} / \mathrm{a}$ & Involved in the study \\
\hline Х & $\square$ ChIP-seq \\
\hline Х & $\square$ Flow cytometry \\
\hline X & MRI-bas \\
\hline
\end{tabular}

Antibodies

Antibodies used

mAb CR3022 is a published antibody with known reactivity to the RBD of SARS-CoV-1 and 2. 1C7 is an unpublished in-house mAb with reactivity to the $\mathrm{N}$ protein of SARS-CoV-1 and 2.

Validation

Both mAbs were validated by binding studies to cells infected with SARS-CoV-2.

\section{Eukaryotic cell lines}

Policy information about cell lines

Cell line source(s)

Sf9, High Five and and Vero.E6 cells were sourced from ATCC. Expi293F cells were sourced from ThermoFisher.

Authentication

No authentication was performed. All expression constructs were Sanger sequenced.

Mycoplasma contamination

The cell lines were not tested for mycoplasma.

Commonly misidentified lines

(See ICLAC register)

No commonly misidentified cell lines were used

\section{Human research participants}

Policy information about studies involving human research participants

Population characteristics

Recruitment

Ethics oversight
Only de-identified samples were used. This is considered non-human subject research. 16 samples were from COVID19 survivors, 109 negative control samples were from a non-COVID19 infected cohort age 20 to 65+

No participants were enrolled. All samples were preexisting.

Alfred Hospital (ID \#280/14) and University of Melbourne (ID \#1442952.1, 1955465.2) Human Research Ethics Committees, under research permit for project TYH2018322 of Helsinki University Hospital Laboratory and by the IRB of the Icahn School of Medicine at Mount Sinai, NY 
